Western University Scholarship@Western

Centre for Decision Sciences and Econometrics

Technical Reports

Economics Working Papers Archive

1986

\title{
Competition for Agency Contracts
}

R. Preston McAfee

John McMillan

Follow this and additional works at: https://ir.lib.uwo.ca/economicscdse_tr

Part of the Economics Commons

Citation of this paper:

McAfee, R. Preston, John McMillan. "Competition for Agency Contracts." Centre for Decision Sciences and Econometrics Technical Reports, 10. London, ON: Department of Economics, University of Western Ontario (1986). 


\section{THE CENTRE FOR DECISION SCIENCES AND ECONOMETRICS}

Competition for Agency Contracts

R. Preston McAfee and John McMillan

TECHNICAL REPORT NO. 10 FEBRUARY 1986

3

Centre For Decision Sciences And Econometrics Social Science Centre The University of Western Ontario London, Ontario N6A 5C2

Departnent of Econemics Library

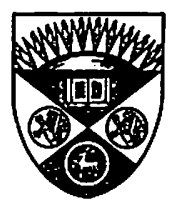

HEB 211986

University of Westem Ontario 
ISSN : $0831-4454$

COMPETITION FOR AGENCY CONTRACTS

\author{
by \\ R. Preston McAfee \\ and \\ John McMillan \\ University of Western Ontario \\ Revised January 1986
}

\begin{abstract}
This paper introduces a market for the services of agents into a principal-agent model. The principal and the potential agents are risk neutral. The contract trades off adverse selection against moral hazard. In a broad range of circumstances, the optimal contract is linear in the outcome. In an incentive-compatible contract, the more able an agent is, the larger is his contractual share of his marginal output; thus a more able agent is induced to work at a rate closer to the first best. Despite the principal's ability to commit himself to a mechanism, the asymetry of information leaves the selected agent with positive surplus.

Correspondence to: J. McMillan

Department of Economics

University of Western Ontario

London, Ontario N6A 5C2

Canada

*

We thank Glenn MacDonald, Paul Milgrom, and Alan Slivinski for useful comments. In a paper developed simultaneously, Laffont and Tirole (1985) derived results which are similar to this paper's.
\end{abstract}

Department of Economics Library

FEB 211986

University of Western Ontario 


\section{INTRODUCTION}

When a principal contracts with an agent for the provision of some good or service, the contract of ten does not specify a fixed price. Instead, the contract contains provisions for price adjustment. For example, when a firm subcontracts some of its input requirements to another firm, or when a government contracts out to a firm the production of a public good, the contract often requires the price to be adjusted in response to unpredictable changes in the supplier's costs. A salesman's remuneration is in part dependent, via commissions, on the amount he sells; but he also receives a fixed sum regardless of how successful he is. The contracts between publisher and author, franchisor and franchisee, landlord and sharecropper, and patent holder and licensee similarly have both fixed and variable components. As Arrow (1985) has noted, "a fee function is a significant departure from the arms-length fixed-price relation among economic agents usually postulated in economic theory" (p. 44). Typically, the fee functions used in practice are simple: contracts involving royalties or commissions make the payment linear in the measured output.

At first glance, it might seem that contractual provisions that lessen the agent's responsibility for his own actions, such as provisions for price adjustment in response to cost fluctuations or fixed payments to salesmen, could not be in the principal's interest. To some extent, the costs incurred or the sales achieved depend upon the effort made by the agent: in other words, there is moral hazard. Such contractual provisions weaken the agent's incentives to act as the principal would want.

The principal-agent literature provides one possible explanation for price-adjustment provisions. If the agent is more risk averse than the 
principal, then it is in their mutual interest to share the burden of the risk. The principal, in designing the contract that is best for him, trades off risk sharing against moral hazard.

often, however, the parties to a contract are large and the risks associated with any one contract are small relative to their overall operations. In such cases, contractual provisions for price adjustment cannot be explained as risk-sharing devices. Indeed, from the point of view of generalized principal-agent theory as envisaged by Myerson (1982), there is no reason why risk sharing should be an inherent part of the principal-agent relationship. What has come to be called the principal-agent model is only one particular model within the broad class of principal-agent models. This paper offers an alternative principal-agent model, applicable when both principal and agent are risk neutral. The model provides an explanation for contractual provisions for price adjustment without appealing to risk aversion. This paper introduces a market for the services of agents. Potential agents compete with each other for the contract with the principal. The potential agents have different types (for example, innate ability or expected production cost) which the principal cannot observe: in other words, there is adverse selection in the market for agents' services. The principal designs a contract which exploits the competition among the potential agents and induces them to reveal their types. Instead of, as in the usual principal-agent model, trading off risk sharing against moral hazard, in this setting the contract trades off adverse selection against moral hazard. Principal-agent models in which there is adverse selection as well as moral hazard have been developed by Holmstrom (1983), Laffont and Tirole (1985, 1986), McAfee and McMillan (1984), and Sappington (1983, 1984). Comparison between these 
models and the present model will be given in the last section of this paper. Arrow (1985), in evaluating principal-agent theory, noted that the principal-agent relationships observed in the real world differ from those predicted by principal-agent theory. "Most importantly, the theory tends to lead to very complex fee functions. It turns out to be difficult to establish even what would appear to be common-sense properties of monotonicity and the like. We do not find such complex relations in reality" (p. 48). Holmstrom and Milgrom (1985) made a similar point. In contrast, it will be shown that the contract which, for the principal, optimally trades off adverse selection against moral hazard can be relatively simple.

In a broad set of circumstances, the predicted contract is linear in the observed outcome. The contract varies with the selected agent's type: in an incentive-compatible contract, the more efficient an agent is, the larger is his contractual share of his marginal output; thus a more efficient agent is induced to work at a rate closer to the first-best. Nevertheless, except when the selected agent has the highest possible efficiency, the contract elicits less than the ideal amount of effort. Despite the principal's ability to commit himself to his mechanism, the asymmetry of information leaves the selected agent with some of the surplus. When the number of potential agents competing for the contract is large enough that there is perfect competition in the market for agents' services, the contract has the selected agent paying a lump sum for the right to do the work and then keeping all of his output.

Thus moral hazard and adverse selection are inherently linked: they cannot be considered in isolation. 


\section{ADVERSE SELECTION AND MORAL HAZARD}

A risk-neutral principal wishes to contract with one agent. There are $\mathbf{n}$ risk-neutral potential agents who compete for the contract. The potential agents have different characteristics, denoted $z$ (measuring, for example, efficiency, or ability, or expected production cost). Only the potential agent himself knows his own type $z$; the other potential agents and the principal perceive types as being independently drawn from a distribution $G(z)$, with density $g(z)$. Thus differences among the potential agents are assumed to reflect inherent differences in their productivity, so that, after learning that he has an unusually high or low $z$, a potential agent has no reason to revise his estimates of the others' $z$ values. (This is the independent-private-values assumption: for a thorough discussion of this assumption, see Milgrom and Weber (1982, pp. 1090-1098).) Let $z_{0}=\sup \{z$ : $G(z)=0\}$ and $z_{1}=\inf \{z: G(z)=1\}$ denote respectively the lowest and highest possible types.

The principal, having announced the contractual payment terms, asks the potential agents to report their types. Denote by $b$ an agent's reported type. On the basis of these reports, he selects one agent.

The agent who wins the contract then takes an action a; assume $a \in(0, \infty)$. The action a can be interpreted as effort. This action together with a random state of nature determines a monetary outcome $\mathbf{x}$ (interpreted as output or sales or production cost). The agent observes this random variable only after he has chosen his action a. The principal cannot observe either the action a or the state of nature; he only observes the outcome $x$. Hence he cannot directly reward the agent for his action. The agent incurs a cost of action which is, in monetary terms, $\phi(a, z)$. The fact that $\phi$ can depend on $z$ 
means that the different potential agents can have different outside opportunities. Assume that (with subscripts denoting partial derivatives) $\phi_{a}>0$ and $\phi_{\text {aa }}>0$.

Because the state of nature is random, the outcome $x$ is a random variable, whose distribution depends upon the agent's type $z$ and action $a$ : the greater the agent's ability $z$, or the larger his effort a, the larger is his output $x$ on average. Denote by $\mu(z, a)$ the expected value of $x$; assume that $0<\mu_{a}<\infty, \mu_{\text {aa }} \leq 0, \mu_{z} \geq 0$.

The principal can make his payment to the agent depend upon the agent's reported type and the observed outcome. Let $s(b, x)$ denote the payment function to which the principal comnits himself. Thus the principal's return is $x-s(b, x)$ and the selected agent's return is $s(b, x)-\phi(z, a)$. The net (social) expected return is $\mu(z, a)-\phi(z, a)$, which is presumed to be positive for some $z, a$. (Note that the risk neutrality of the potential agents means that the principal gains nothing by making the payment to the selected agent a function of the other potential agents' reports, or by requiring payment from the other potential agents: compare with Harris and Raviv (1981), Myerson (1981), and Riley and Samuelson (1981). Thus this is an optimal mechanism for the principal.)

Three assumptions on the technology are imposed. First, suppose that the probability distribution function of the outcome $x$ can be expressed as $F(x, h(z, a))$ for some continuously differentiable function $h$. The weak separability here allows the agent to dissimulate: it implies that, with enough effort $a$, an agent can make his ability $z$ appear to be slightly lower or higher than it actually is. This implies that $\mu_{z} / \mu=\underset{z}{h} / \mathrm{h}$. It also 
implies that

$$
\frac{\partial}{\partial z} \operatorname{ES}(x, b)=\frac{\partial}{\partial a} \operatorname{ES}(x, b) \frac{z}{h}=\frac{\partial}{\partial a} \operatorname{ES}(x, b) \frac{z^{\mu}}{\mu_{a}} .
$$

This assumption was originated by Laffont and Tirole (1986), who defined the "concealment set" to be the set of reports $b$ and actions a such that, even though the agent is actually of type $z$, the distribution of outputs is the same as if he were actually of type $b$.

The assumption that the distribution of outcomes can be represented as $F(x, h(z, a))$ is crucial for the simplicity of the contracts predicted by this model. With a more general production technology, the principal would choose a contract which in part sought to infer from the observed outcome $x$ the agent's true type $z$. The predicted contract would depend in a complex way on the distribution $F$. With the present assumption, the principal cannot hope to infer anything about the type $z$ from the observation $x$ : the agent is able to sabotage, by his choice of action a, any such inferential purpose of the contract. This forces the contract to be relatively simple; indeed it will be shown that the chosen contract depends upon only the first moment of $F$. (Holmstrom and Milgrom (1985) made a similar general point: giving the agent more freedom of action reduces the principal's range of choices and may result in a simple contract being optimal for the principal.)

The second assumption is a single-crossing property (compare, for example, with Maskin and Riley (1984)):

(2) $\phi_{z}<\frac{a}{\mu} \mu_{z}$.

Suppose we hold the distribution of $x$ constant as we vary $z$ (that is, we move 
along a particular $\mu-$ isoquant in a-z space). Then $d a / d z=-\mu_{z} / \mu_{a}$. Then (2) implies $d \phi /\left.d z\right|_{\mu=\text { constant }}<0$. Thus a less able agent must incur a higher cost than a more able agent in order to produce a given expected outcome.

Third, assume

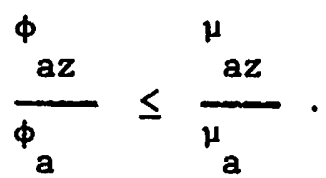

The efficient effort level satisfies $\mu_{a}=\phi_{a}$. Condition (3) implies that

(4)

$$
\left.\frac{\mathrm{da}}{\mathrm{dz}}\right|_{\mu_{\mathrm{a}}}=\phi_{\mathrm{a}}=-\frac{\frac{\mu_{\mathrm{az}}}{\mu_{a}}-\frac{\phi_{a z}}{\phi_{a}}}{\frac{\mu_{\mathrm{aa}}}{\mu_{a}}-\frac{\phi_{a a}}{\phi_{a}}} \geq 0 .
$$

That is, the efficient level of effort is nondecreasing in type; or, more intuitively, an agent with greater ability has a larger marginal product of effort.

This problem involves both adverse selection (since the principal cannot observe the potential agents' types $z$ ) and moral hazard (since the principal cannot observe the selected agent's action a). The purpose of this paper is to derive the principal's optimal payment function $s(b, x)$.

\section{THE AGENT'S CHOICE OF ACTION}

In this section we shall examine the contract offered by the principal in terms of the action a that an agent of type $z$ chooses; in terms, that is, of the function $a(z)$. We show that the function $a(z)$ can be used to characterize how much the agent is paid, without specifying the contract. 
This corresponds to the generalized principal-agent formulation of Myerson (1982), in which the principal recommends a decision $a(z)$ to the agent of type $z$, and the agent finds it in his interest to obey this recommendation.

Consider first the optimizing actions of the potential agents. As Myerson (1982) proved, by the Revelation Principle we can, without loss of optimality, presume that the potential agents reveal their types honestly to the principal. (Although Myerson's proof applies to the case of a finite set of types, the same method of proof extends to the continuum case.) Thus the principal will optimally select the agent who reports the highest type; and $G(b)^{n-1}$ is the probability that a potential agent who reports his type to be $b$ wins. The ex ante expected utility of a potential agent of type $z$ who reports his type to be $b$ is therefore

(5) $\pi(b, a, z)=[E S(b, x)-\phi(z, a)][G(b)]^{n-1}$.

The incentive-compatibility condition, to be further examined in the next section is

(6) $\pi(b, a, z) \leq \pi(z, \hat{a}, z)$,

where $\hat{a}$ is the agent's optimal action given that he reports $z$. That is, $\hat{a}$ satisfies $\pi_{a}=0$ or

(7) $\frac{\partial}{\partial a} \operatorname{ES}(z, x)=\phi_{a}$.

Using (1), (7) can be rewritten as

(8) $\frac{\partial}{\partial z} \operatorname{ES}(z, a)=\frac{\phi_{a}}{\mu_{a}} \mu_{z}$.

(Note that (7) presumes that the contract chosen by the principal satisfies $\partial^{2} \mathrm{ES} / \partial \mathrm{a}^{2} \leq \phi_{\mathrm{aa}}$. It will be seen in the next section that the optimal linear contract satisfies this condition.) 
Each potential agent is assumed to have freedom of exit, so that expected profits must be nonnegative. (Note that, since the cost of effort $\phi$ may depend on $z$, it is possible for the potential agents to have different alternative opportunities which vary with their abilities $z_{.}$)

The first result characterizes the selected agent's optimizing choice of action. (The proofs of all of the following results are in the Appendix.) Lemma 1: Let $V(z, a)=\operatorname{ES}(z, x)-\phi(z, a)$ represent the expected profit of the selected agent given that he reports $b=z$. Then, with the agent choosing his action $a(z)$ optimally, his expected profit is

$$
V(z, a(z))=G(z))^{-(n-1)} \int_{0}^{z}\left[\phi_{a}(s, a(s)) \frac{z^{\mu(s, a(s))}}{\mu_{a}(s, a(s))}-\phi_{z}(s, a(s))\right] G(s)^{n-1} d s .
$$

Consider now the principal's optimizing choice of contract, given that the agent will respond as implied by Lemma 1 .

Lemma 2: Let $\mathbf{z}$ be the highest reported type. Define

$$
A(z, a)=\mu(z, a)-\phi(z, a)-\frac{1-G(z)}{g(z)}\left[\frac{a^{a}(z, a)}{\mu_{a}(z, a)} \mu_{z}(z, a)-\phi_{z}(z, a)\right] \text {. }
$$

If $A(z, a) \geq 0$ for some $a$, then the principal desires to evoke from the agent an action $a^{*}(z)$ given by

$$
a *(z)=\underset{a}{\arg \max _{a} A(z, a) .}
$$

If $A(z, a)<0$ for all a, the principal refuses to contract with any of the agents. 
Note three features of the level of action which the principal wishes to evoke from the selected agent.

First, the efficient action is that which maximizes $\mu(z, a(z))-$ $\phi(z, a(z))$. But

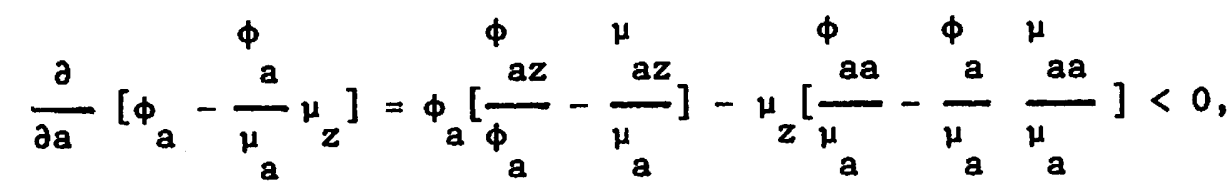

the inequality following from (3). Hence

$$
\mu_{a}\left(z, a^{*}(z)\right)-\phi_{a}\left(z, a^{*},(z)\right)>0 \text { if } z<z_{1} .
$$

It follows that, for $z<z_{1}$, a less than efficient amount of effort is obtained. If the selected agent has the highest possible level of ability, so that $z=z_{1}$, then the first-best effort level is achieved (that is, $\mu_{a}=\phi_{a}$ ). Second, the desired action $a^{\star}(z)$ is invariant to $\mathrm{n}$ : the size of the inefficiency is not affected by the extent of competition among the potential agents (except to the extent that the more potential agents there are, the higher the selected agent's type $z$ is likely to be).

Third, the possibility that no contract is awarded (if $A(z, a)<0$ ) acts like a reserve price in an auction (Harris and Raviv (1981), Laffont and Maskin (1980), Milgrom and Weber (1982), Myerson (1981), Riley and Samuelson (1981)): the principal introduces this possibility to stimulate competition among the potential agents. To see this, note that the expected value of $[1-G(z)] / g(z)$ is the expected difference between the highest type and the second highest type (by the usual auction-theory intuition). From the discussion following equation (2), $\left[\left(\phi_{a} \mu_{z} / \mu_{a}\right)-\phi_{z}\right]$ is equal to $\mathrm{d} \phi /\left.\mathrm{dz}\right|_{\mu=\text { constant. That is, it measures the rate at which the cost } \phi \text { of }}$ maintaining a given average outcome $\mu$ increases as type $z$ decreases. Hence 
the third term on the right side of (10) shows the expected extra production cost of producing a fixed output if the second-best potential agent is chosen instead of the best potential agent. Note that from (10), it is possible that $A(z, a)<0$ even though $\mu(z, a)-\phi(z, a)>0$ for some a: that is, no contract is awarded although there are ex post gains from trade. Thus, as in the usual auction model, the reserve price introduces the possibility of an inefficient outcome.

Throughout this section it was assumed that there is an interior solution for the action a; that is, the optimal action a* prescribed by (11) is positive. Should (11) imply a negative $a^{*}$, then clearly the optimum is the corner solution $a=0$. This caveat likewise applies in what follows.

\section{LINEAR CONTRACTS}

Given the constraints imposed upon the principal by the informational asymmetry, the best level of action he can elicit from the agent of type $z$ is $a^{*}(z)$. Hence the principal has solved his optimization problem if he can find an incentive-compatible contract eliciting the action $a^{*}(z)$. The main result of this paper shows that, in many cases, a contract which is linear in x suffices.

Theorem 3: The principal's optimal contract is linear in $x$ for any number of potential agents $n$ if and only if

$$
\frac{d}{d z} \frac{a^{\left(z, a^{*}(z)\right)}}{\mu_{a}\left(z, a^{*}(z)\right)} \geq 0,
$$

where $a^{*}(z)$ is defined by (11). If so, the optimal contract is

$$
s(b, x)=\beta(b)+\alpha(b) x,
$$

where 


$$
\alpha(b)=\frac{\phi_{a}\left(b, a^{*}(b)\right)}{\mu_{a}\left(b, a^{*}(b)\right)}
$$

and

(17)

$$
\begin{aligned}
& \beta(b)=-\frac{\phi_{a}\left(b, a^{*}(b)\right)}{\mu_{a}\left(b, a^{*}(b)\right)} \mu\left(b, a^{*}(b)\right)+\phi\left(b, a^{*}(b)\right) \\
& +G(b)^{-(n-1)} \int_{z}^{b}\left[\frac{\phi_{a}\left(s, a^{*}(s)\right)}{\mu\left(s, a^{*}(s)\right)} \mu_{z}\left(s, a^{*}(s)-\phi_{z}\left(s, a^{*}(s)\right)\right] G(s)^{n-1} d s .\right.
\end{aligned}
$$

The proof (in the Appendix) first shows that, if a contract is linear, it must have the form given in the theorem. Then it shows that the agent will select an action $\hat{a}$ which is a function of both his reported type $b$ and his true type z. Finally, it shows that, given the condition (14), the contract (15) induces any potential agent to report his type $z$ honestly.

The contract described in Theorem 3 is not the only optimal contract: there are nonlinear contracts which evoke the same level of effort. The point of Theorem 3 is that in certain circumstances it is possible for the principal to achieve his optimum by using a linear contract.

For a contract linear in outcome $x$ to be optimal for the principal, the sharing term $\alpha$ must increase with type $z$. In particular, a more able agent faces a larger $\alpha$ and thus bears a greater responsibility for his own actions. Incentive compatibility is the reason why $\alpha$ must increase with $b$. If $\alpha$ increases with $b$, then it does not pay a potential agent with low ability to claim he is more able than he really is, because he is then 
penalized by having a contract which makes his payment sensitive to his output; since he actually has low ability, his output, and therefore his payment, will be low. The same argument in reverse shows why an able potential agent would not claim to have low ability when $\alpha$ increases with $b$. An unsurprising corollary of the theorem is that, since the contract allows a more able agent to keep a larger share of his marginal output, a more able agent will work harder.

Corollary 4: If $\mathrm{d} \alpha / \mathrm{dz} \geq 0$ then $\mathrm{da}(\mathrm{z}) / \mathrm{dz} \geq 0$.

The sharing term $\alpha$ satisfies $\alpha \mu_{a}=\phi_{a}$. This has the common-sense interpretation that the agent equates his marginal cost of effort, $\Phi_{a}$, to the expected marginal benefit that accrues to him (since the agent keeps a fraction $\alpha$ of the expected output $\mu$ ).

To illustrate these results, and in particular to help understand the necessary and sufficient condition (14) for an optimal contract to be linear, consider an example. Suppose $\mu(z, a)=z+a$ and $\phi(z, a)=\delta a^{2} / 2$. It is easy to check that these functional forms satisfy the assumptions stated in Section 2.

From (11), the action desired by the principal is

$$
a *=\operatorname{argmax}\left\{z+a-\frac{\delta a^{2}}{2}+\frac{1-G(z)}{g(z)}[-a \delta]\right\}
$$

Differentiation with respect to a yields

$$
a *=\frac{1}{\delta}-\frac{1-G(z)}{g(z)} \text {. }
$$


Compare this with the efficient action (which satisfies $\mu_{a}=\phi_{a}$ ): this is equal to $1 / \delta$. Hence the difference between the equilibrium outcome and what would be attained were it not for the informational asymetries is the inverse hazard rate $(1-G(z)) / g(z)$.

The contract parameters are (from (16) and (17))

$$
\alpha(b)=\delta a *=1-\delta\left(\frac{1-G(b)}{g(b)}\right)
$$

and

$$
\begin{aligned}
& \beta(b)=-\left[1-\frac{\delta(1-G(b))}{g(b)}\right]\left[b+\frac{1}{\delta}-\frac{1-G(b)}{g(b)}\right] \\
& +\frac{\delta}{2}\left(\frac{1}{\delta}-\frac{1-G(b)}{g(b)}\right)^{2}+G(b)^{-(n-1)} \int_{z}^{b}\left(1-\delta \frac{(1-G(s))}{g(s)}\right) G(s)^{n-1} d s .
\end{aligned}
$$

The necessary and sufficient condition for implementability by a linear contract, $d\left(\phi_{a} / \mu_{a}\right) / d z \geq 0$, reduces to

$$
\frac{d}{d z}\left[\frac{1-G(z)}{g(z)}\right] \leq 0,
$$

which is monotonic-inverse-hazard-rate condition familiar from auction theory and elsewhere (Myerson (1981)). Note that, from (19), (22) implies that the deviation of equilibrium effort from the first-best level declines as ability $z$ increases.

\section{NONLINEAR CONTRACTS}

We digress now to examine the nature of the contract when the principal is forced to use a nonlinear contract because (14) is not satisfied. The advantage of a nonlinear contract is that it allows the principal to use more of his knowledge about the distribution of $x$ in inducing the agent to choose 
the desired action $a^{*}(z)$.

For the purposes of illustration, we consider only the extreme case in which the outcome $x$ is nonstochastic: thus $x=\mu(z, a)$. The principal can, in this case, condition payment on the choice of effort: thus he pays the agent only when $a=a *(b)$ given the agent's report $b$. This forces an agent of type $z$ who reported his type to be $b$ to choose an effort $\hat{a}$ satisfying $\mu\left(b, a^{*}(b)\right)=\mu(z, \hat{a})$. Thus

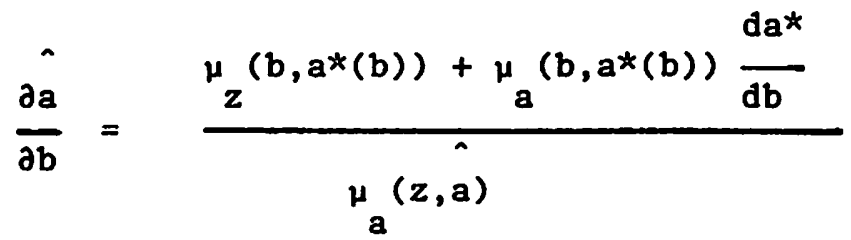

and

(24)

$$
\frac{\partial a}{\partial z}=-\frac{z^{\mu(z, \hat{a})}}{\mu_{a}(z, \hat{a})} .
$$

\section{Theorem 5: Incentive compatibility is satisfied regardless of the number of} potential agents $\mathrm{n}$ if and only if

$$
\frac{d}{d z} \mu\left(z, a^{\star}(z)\right) \geq 0 .
$$

It follows from this result that there is an incentive--compatible contract which implements the action desired by the principal, $a^{\star}(z)$, if and only if 
(26) $\quad \frac{d a^{*}}{d z} \geq-\frac{{ }^{\mu}}{\mu a}$

Thus, in general, there will be desired actions $a^{\star}(z)$ that the principal can implement by using nonlinear contracts but not by using linear contracts.

(Compare with Corollary 4 above, noting that $-\mu_{z} / \mu_{a}<0$. )

Finally, note that the assumption of a nonstochastic $x$ is not as extreme as it might appear. Suppose that $F_{x}(x, h(z, a))$ has a mode at a point $\tilde{x}(h(z, a))$, and $\tilde{x}$ is monotonic in $h$. Suppose the principal offers the payment scheme

$$
s(z, a)= \begin{cases}p(\varepsilon), & \mid x-\tilde{x}\left(h\left(b, a^{*}(b)\right) \mid<\varepsilon\right. \\ 0, & \text { otherwise }\end{cases}
$$

where

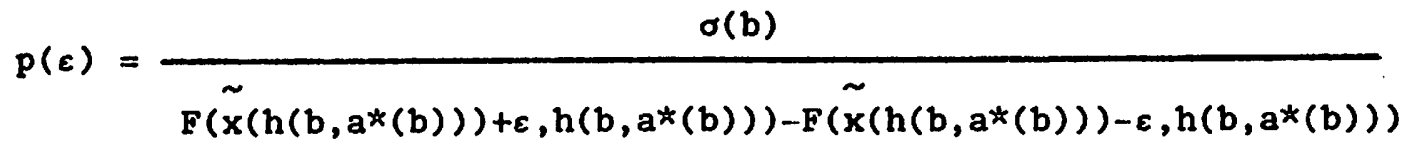

where $\sigma(b)$ is the payment function in the nonstochastic case (that is, $\sigma(b)=$ $G(b)-(n-1) \pi(b, \hat{a}, z)$ as in equation (A18). Then the principal can approximate arbitrarily closely the outcome of the nonstochastic case by letting $\varepsilon \rightarrow 0$. of course this result is very sensitive to the risk neutrality of the agent and is not considered further, except to note that the uncertainty of the output strengthens the case for using a linear contract where possible, as the linear contract requires the principal to know only the mean and not the shape of the distribution $F$. 


\section{PROPERTIES OF THE LINEAR CONTRACT}

Return now to considering the optimal linear contract. What predictions does this model make about the form of the principal-agent contract that will be observed when there is a market for the services of agents?

The striking feature of the contract is that, in a broad range of circumstances, it is linear. Linear contracts have obvious practical advantages in ease of use. For this reason, and because of the analytical simplicity of linear contracts, contracting models sometimes impose linearity as an ad hoc assumption (see for example McAfee and McMillan (1984), Stiglitz (1974), and weitzman (1980)). This paper shows that there are cases in which the optimal contract is linear in the outcome (although it is not linear in the agent's ability). Payment schemes used in practice which involve royalties or comissions are linear in the outcome.

Further simplicity comes from the fact that the only knowledge about the probability distribution of outputs that the principal needs is how average output varies with ability and effort. The optimal contract does not require the principal to have any more precise, detailed knowledge of the distribution of outputs than the mean.

An agent who reports his ability level to be $b$ and is selected is paid an amount $\beta(b)+\alpha(b) x$ in exchange for his output $x$. The lump-sum term $\beta$ (b) can be interpreted as a salary or fixed payment; the sharing term $\alpha(b)$ can be interpreted as a royalty or commission rate or an incentive scheme. Although $\alpha(b)$ and $\beta(b)$ depend upon the agent's reported ability, they are fixed for the selected agent. The competing potential agents in effect are offered a choice of contracts, knowing that the potential agent who picks the largest sharing term $\alpha$ (b) will win the contract. Thus each potential agent 
is induced to reveal his level of ability by his choice of contract.

The sharing term $\alpha(b)$ satisfies $0 \leq \alpha(b) \leq 1$. The sharing term

$\alpha$ (b) increases with the agent's ability. Thus the more able an agent is the larger the fraction of his marginal output he is allowed to keep. As a result, a more able agent is induced to work harder.

If the agent has the highest possible ability, then the principal sets his sharing term equal to unity. (From $(10)$ and $(16), \alpha\left(z_{1}\right)=1$.) Thus the most able possible agent keeps all of his marginal output, and works at his first-best rate.

The size of the sharing term $\alpha(b)$ and the resultant amount of effort extracted from the agent are independent of the number of competing potential agents (although as the number of potential agents rises, the expected highest ability level rises and, for this reason, the expected sharing term $\alpha(b)$ rises). In particular, the analysis applies to the case of $n=1$ : the principal still faces a trade off between adverse selection and moral hazard even when there is no competition for the contract. Since the sharing term $\alpha(b)$ is independent of the number of potential agents, increasing the amount of competition simply has the effect ceteris paribus of shifting downwards the intercept term, $\beta(b)$.

Recall that the contract can be written as (from (15), (16), and (17))

$$
\begin{aligned}
& s(z, x)=\frac{\phi\left(z, a^{*}(z)\right)}{\mu a^{\left(z, a^{*}(z)\right)}\left[x-\mu\left(z, a^{*}(z)\right)\right]+\phi\left(z, a^{*}(z)\right)} \\
& +G(z)^{-(n-1)} \int_{z}^{z}\left[\frac{a^{\mu}\left(s, a^{*}(s)\right)}{\mu\left(s, a^{*}(s)\right)} \mu_{z}\left(s, a^{*}(s)\right)-\phi_{z}\left(s, a^{*}(s)\right)\right] G(s)^{n-1} d s .
\end{aligned}
$$

Consider the expected payment to the agent, $\operatorname{ES}(z, x)$. The expected value of the first term on the right side of (29) is zero (since by definition $\mu=E x$ ). 
The second term is the cost incurred by the agent. Hence the third term is the agent's expected profit.

As the market for agents' services becomes perfectly competitive $(n \rightarrow \infty)$, the selected agent's expected profit goes to zero. In the limit, with an infinity of potential agents, with probability one the selected agent has the highest possible ability $z_{1}$. In this case the agent keeps all of his marginal output. Then $\beta=\phi-\mu$. Since $\mu>\phi, \beta$ is negative: the agent pays for the right to do the work. The size of this payment is such that, in expectation, the agent produces just enough to cover his initial payment to the principal of $\beta$.

The fixed payment $\beta$ may be positive or negative; it is monotonically decreasing in the number of competing potential agents $n$. It will tend to be positive when the number of potential agents is small and the selected agent has low ability; and it will tend to be negative when there is much competition and a relatively able agent is selected.

The payment per unit of output is $\alpha+\beta / x$. Unless $\beta=0$ (a measure zero event), the contract has the unit price of the agent's output adjusting with the realized output. Alternatively, the model can be interpreted to represent a contract for the supply of one unit of some good or service. Then $x$ represents production cost, and the contract price is predicted to vary with realized production cost. Hence the model predicts that contracts will contain provisions for price adjustment (compare with the discussion in the introduction). 


\section{COMPARISONS WITH THE LITERATURE}

As noted by Arrow (1985) in the paper quoted in the introduction, the usual principal-agent model, with a trade-off between risk sharing and moral hazard, predicts complicated contracts. Little can be said about the form of the contract or how much effort it induces the agent to make. (See Harris and Raviv (1979), Holmstrom (1979), Shavell (1979), MacDonald (1984)). In contrast, the present model, trading off adverse selection against moral hazard, predicts that, in a wide range of circumstances, the contract will be linear. (It should be stressed, however, that this result depends upon the assumptions stated in Section 2; in particular, conditions (1), (2), and (3).) Moreover, as described in the previous section, the model has several potentially testable predictions. Also, in the usual principal-agent model, the principal is able to extract all of the surplus, despite the asymmetry of information. In the present model, the adverse selection results in the agent being left with positive surplus.

A direct comparison between the present model and the usual principal-agent model is obtained by noting that, relative to the usual model, the present model includes an additional random shock, specific to each of the potential agents, and observed by the potential agent before he agrees to the contract.

Holmstrom and Milgrom (1985) showed that changing the usual principal-agent model to give the agent greater freedom of action (by introducing multidimensional actions or actions over time) generates, in a special model, linear contracts. In a broad sense, the approach of this paper is similar. The simplicity of this model's contract follows from the assumption, also used by Laffont and Tirole $(1985,1986)$, that the 
distribution of outcomes can be expressed as $F(x, h(z, a))$ so that the principal can infer nothing about the agent's type $z$ from observing the outcome $x$ : an agent with low ability is locally able to imitate undetectably a more able agent.

Holmstrom (1983) and Sappington (1983, 1984) showed that the combination of adverse selection and moral hazard often leads to an inefficient outcome despite the risk neutrality of the agent. Laffont and Tirole (1986) analyzed the case of a single agent with adverse selection and showed that the contract is linear in the agent's type. Laffont and Tirole (1985) analyzed competition for contracts in a model similar to the present model, except that the payment to the winning bidder depends not only on the winning bidder's reported ability but also on the second-highest reported ability: this scheme has the advantage that, like the vickrey auction, the bidding game has a dominant equilibrium. McAfee and McMillan (1984) modelled the trade-off between adverse selection and moral hazard in a second-best sense: the contract is by assumption linear in both ability and output (although the analysis there is more general than the present analysis in allowing the potential agents to be risk averse). Riley (1985) showed, in the context of a general auction model but without moral hazard, that it is in the seller's interest to require payment to depend not only on the bids but also on any ex post information he has which is correlated with the successful bidder's true valuation of the item: in the context of the present model, this says that, as seen above, the payment should be a function of output $x$ as well as report $b$. 


\section{APPENDIX}

Proof of Lemma 1: Note first that $V\left(z_{0}, a\right)=0$. This is because free exit guarantees that profits are nonnegative, or $V\left(z_{0}, a\right) \geq 0$. Suppose $v\left(z_{0}, a\right)>$ 0 . Then for small $\delta, \pi\left(z_{0}+\delta, a, z_{0}\right)>0=\pi\left(z_{0}, a, z_{0}\right)$, contradicting incentive compatibility.

Now, using the Envelope Theorem, (5), and (8),

(A1) $\frac{d \pi}{d z}=\frac{\partial \pi}{\partial z}=\left[\left.\frac{\partial}{\partial z} \operatorname{ES}(b, x)\right|_{b=z}-\phi_{a}(z, a)\right] G(z)^{n-1}$

$$
\left.=\phi_{a}(z, a(z)) \frac{z^{(a, a(z))}}{\mu_{a}(z, a(z))}-\phi_{z}(z, a(z))\right] G(z)^{n-1} .
$$

Integrating from $z_{0}$ to $z$, using $V\left(z_{0}, a\right)=0$ and (5), proves the result. QED.

Proof of Lemma 2: The principal's expected profit is

$$
\text { (A2) } \begin{aligned}
& \Pi=\int_{z}^{z}[\mu(z, a(z))-E S(z, x)] n G(z)^{n-1} g(z) d z \\
&=\int_{z}^{z}\left[\mu(z, a(z))-(\phi(z, a(z))+V(z, a(z))] n G(z)^{n-1} g(z) d z\right. \\
&=\int_{0}^{1}\left[\mu(z, a(z))-\phi(z, a(z))-\frac{1-G(z)}{g(z)} \frac{\phi_{a}(z, a(z))}{\mu(z, a(z))} z_{a}(z, a(z)) .\right. \\
&-\phi(z, a(z)] n G(z)^{n-1} g(z) d z,
\end{aligned}
$$

the second line following from the definition of $V$ and the third line following from (9) and integration by parts. If $A(z, a)<0$ for all $a$, then clearly the principal earns negative expected profit and so does not contract with any agent. Otherwise, the agent's best action, from the principal's point of view, satisfies (11). 
Proof of Theorem 3: Consider first the implications of the contract's linearity. Suppose $s(b, x)$ is of the form (15). Then

(A3) $\quad \operatorname{ES}(b, x)=\beta(b)+\alpha(b) \mu(z, a)$.

From (7),

(A4)

$$
\phi_{a}(z, a)=\alpha(b) \underset{a}{\mu_{a}}(z, a),
$$

which implies (16). Thus, from Lemma 1 ,

(A5) $\beta(z)+\alpha(z) \mu(z, a)-\phi(z, a)=G(z)^{-(n-1)} \int_{z}^{z}\left[\frac{a}{\mu} \mu_{z}-\phi_{z}\right] G(s)^{n-1} d s$,

which implies

$$
\text { (A6) } \begin{aligned}
\beta(b)= & G(b)-(n-1) \int_{z}^{b}\left[\frac{a(b, a(b))}{\mu(b, a(b))} \mu_{z}(b, a(b))-\phi_{z}(b, a(b)] G(s)^{n-1} d s\right. \\
& -\alpha(b) \mu(b, a(b))+\phi(b, a(b)) .
\end{aligned}
$$

Consider now the agent's choice of action as a function both of his type $z$ and his report $b$ (which need not equal $z$ ). From (5), (15), (16), and (A6),

$$
\text { (A7) } \begin{aligned}
\pi(b, a, z) & =\int_{z}^{b}\left[\frac{a}{\mu(b, a(b))} \mu_{z}(b, a(b))-\phi_{z}(b, a(b))\right] G(s)^{n-1} d s \\
& +G(b)^{n-1}\left[\frac{a(b, a(b))}{\mu(b, a(b))}(\mu(z, a)-\mu(b, a(b))+\phi(b, a(b))\right. \\
& -\phi(z, a)] .
\end{aligned}
$$

Differentiate (A7) with respect to the variable a:

$$
\therefore \quad(A 8) \quad \pi_{a}(b, a, z)=G(b)^{n-1}\left[\frac{a^{(b, a(b))}}{\mu(b \cdot a(b))} \mu_{a}^{\mu}(z, a)-\phi_{a}(z, a)\right] \text {. }
$$


(A9) $\pi_{a a}(b, a, z)=G(b)^{n-1}\left[\frac{a^{\mu(b, a(b))}}{\mu_{a}^{(b, a(b))}} \mu_{a a}(z, a)-\phi_{a a}(z, a)\right]<0$.

Thus, for any $(b, z)$, the agent chooses a unique $a=$ a satisfying

$$
\frac{\phi_{a}(b, a(b))}{\mu_{a}(b, a(b))}=\frac{\phi_{a}(z, a)}{\mu_{a}(z, a)}
$$

Hence, the chosen action a varies with both $b$ and $z$.

Finally, consider the incentive compatibility of the agents' reports.

Note that $\pi_{b}(z, \hat{a}, z)=0$ together with $\pi_{b z}(b, \hat{a}, z) \geq 0$ are necessary for incentive compatibility; that is for $b=z$ to be an optimal report for $a$

potential agent. (This is because $0=d \pi_{b}(z, \hat{a}, z) / d z=\pi_{b b}(z, \hat{a}, z)+$ $\pi_{b z}(z, \hat{a}, z)$, which implies $\pi_{b z}(z, \hat{a}, z) \geq 0$ is necessary. $)$ In addition, $\pi_{b}(z, \hat{a}, z)=0$ and $\pi_{b z}(b, \hat{a}, z) \geq 0$ are sufficient for incentive compatibility.

(This is because $\pi b z(b, a, z) \geq 0$ implies $\pi b(b, z) \stackrel{\geq}{<} 0$ as $b \frac{\leq}{>} z$, which means that $\pi_{b z}(b, \hat{a}, z) \geq 0$ is sufficient. $)$

It remains therefore to evaluate $\pi_{b}(z, \hat{a}, z)$ and $\pi_{b z}(b, \hat{a}, z)$. From (A7),

$$
\text { (AI1) } \begin{gathered}
\pi_{b}(b, \hat{a}, z)=G(b){ }_{\left\{\frac{a}{\mu}(b, a(b))\right.}^{n-1} \mu_{z}(b, a(b))-\phi_{z}(b, a(b)) \\
+\alpha^{\prime}(b)[\mu(z, a)-\mu(b, a(b))] \\
-\frac{\phi_{a}(b, a(b))}{\mu_{a}(b, a(b))}\left[\mu_{z}(b, a(b))-\mu_{a}(b, a(b)) G^{\prime}(b)\right]+\phi_{z}(b, a(b)) \\
+\phi_{a}(b, a(b)) a^{\prime}(b)
\end{gathered}
$$




$$
\begin{aligned}
\left.\left.+\frac{(n-1) g(b)}{G(b)}[\alpha(b)(\mu(z, a)-\mu(b, a(b)))+\phi(b, a(b))-\phi(z, a)\}\right\}\right\} \\
= \\
+\frac{\left(b ( b ) ^ { n - 1 } \left\{\alpha^{\prime}(b)[\mu(z, a)-\mu(b, a(b))]\right.\right.}{G(b)}[\alpha(b)(\mu(z, a)-\mu(b, a(b)))+\phi(b, a(b)) \\
\quad-\phi(z, a)]\} .
\end{aligned}
$$

Hence, since $\hat{a}(z, z)=a^{*}(z), \pi_{b}(z, \hat{a}, z)=0$.

Now differentiate (A11):

(A12) $\pi_{b z}(b, a, z)=G(b){ }^{n-1}\left[\frac{d \alpha}{d b} \frac{d \mu(z, a)}{d z}+\frac{(n-1) g(b)}{G(b)}\left(\alpha(b) \mu_{z}-\phi_{z}\right)\right]$

$\therefore$

since, at $a, \frac{\partial}{\partial a}[\alpha(b) \mu(z, a)-\phi(z, a)]=0$. Note that since, from (A10), $\alpha(b)=\phi a(z, a) / \mu a(z, a)$, it must be that $\alpha(b) \mu z-\phi z \geq 0$.

Therefore, since $\pi_{b z}$ must be nonnegative for all $n$ including $n=1,(A 12)$ implies

(A13) $\frac{d \alpha}{d b} \frac{d \mu(z, a)}{d z} \geq 0$.

But

$$
\text { (A14) } \frac{d \mu(z, a)}{d z}=\mu_{z}+\mu \frac{d a}{a} \frac{\hat{d}}{d z} \text {; }
$$

hence $d \mu / d z \geq 0$ if $\hat{d a} / d z \geq 0$. But, from (A10),

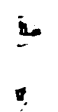




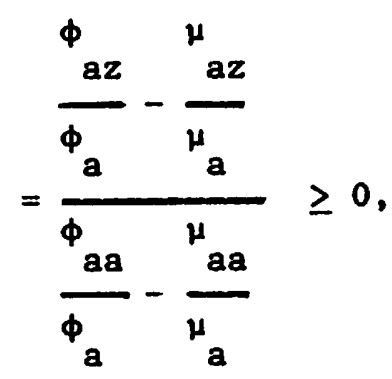

the inequality following from (3). Hence $\pi_{b z}(b, \hat{a}, z) \geq 0$, so that $b=z$ is the global optimum.

QED

Proof of Corollary 4: From (16)

$$
\begin{aligned}
& \text { (A16) } \frac{d \alpha(z)}{d z}=\frac{\phi_{a z}}{\mu_{a}}-\frac{a^{\mu} a z}{\mu_{a}^{2}}+\frac{d a(z)}{d z}\left[\frac{a a}{\mu}-\frac{a}{\mu} \frac{a}{a}{ }_{a}^{\mu}\right] \\
& =\alpha(z)\left[\frac{\phi_{a z}}{\phi_{a}}-\frac{\mu_{a z}}{\mu_{a}}+\frac{d a(z)}{d z}\left[\frac{\phi_{a a}}{\phi_{a}}-\frac{{ }_{a a}}{\mu_{a}}\right]\right] .
\end{aligned}
$$

Hence $\mathrm{d} \alpha / \mathrm{d} z \geq 0$ implies

$$
\text { (A17) } \frac{\mathrm{da}(\mathrm{z})}{\mathrm{dz}} \geq-\frac{\frac{\phi_{a z}}{\phi_{a}}-\frac{{ }_{a z}}{\mu_{z}}}{\frac{\phi_{a a}}{\phi_{a}}-\frac{{ }_{a a}}{\mu_{a}}} \geq 0,
$$

the last inequality following from (4).

QED.

Proof of Theorem 5: As in the proof of Theorem 3, if $\pi_{b}(z, \hat{a}, z)=0$ then $\pi_{b z}(z, \hat{a}, z) \geq 0$ is necessary and $\pi_{b z}(b, \hat{a}, z) \geq 0$ is sufficient for incentive compatibility.

From Lemma 1, the expected profit earned by an agent choosing action $\hat{a}$ is 
(A18) $\pi(b, \hat{a}, z)=G(b)^{n-1}[\phi(b, a *(b))-\phi(z, a)]+\int_{z}^{b}\left(\phi \frac{z}{\mu}-\phi z\right) G(s)^{n-1} d s$.

Thus

$$
\begin{aligned}
\text { (A19) } & \pi_{b}(b, a, z)=G(b){ }^{n-1}\left\{\phi_{z}\left(b, a^{*}(b)\right)+\phi_{a}\left(b, a^{*}(b)\right) \frac{d a^{*}}{d b}-\phi_{a}(z, a) \frac{\partial a}{\partial b}\right. \\
+ & \phi_{a}\left(b, a^{*}(b)\right) \frac{\mu_{z}\left(b, a^{*}(b)\right)}{\mu_{a}\left(b, a^{*}(b)\right)}-\phi_{z}\left(b, a^{*}(b)\right) \\
+ & \left.\frac{(n-1) g(b)}{G(b)}\left[\phi\left(b, a^{*}(b)\right)-\phi(z, a)\right]\right\} .
\end{aligned}
$$

Thus, using (23), $\pi_{b}(z, \hat{a}, z)=0$.

A-
$\therefore$

$$
\begin{aligned}
& \text { (A20) } \pi_{b z}(b, \hat{a}, z)=G(b)^{n-1}\left\{-\left(\frac { d } { d b } \mu ( b , a * ( b ) ) \left(\frac{d}{d z} \frac{\phi_{a}(z, a)}{\mu_{a}(z, a)}\right.\right.\right. \\
& \left.-\frac{(n-1) g(b)}{G(b)}\left(\frac{d}{d z} \phi(z, \bar{a})\right)\right\} .
\end{aligned}
$$

But

$$
\text { (A21) } \frac{d}{d z} \phi(z, \hat{a})=\phi_{z}-\phi_{a} \frac{z}{\mu}<0
$$

and

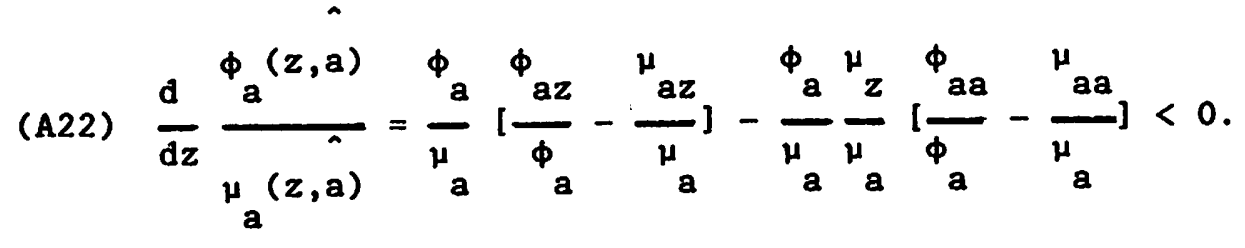

This condition must be satisfied for all $n$; in particular for $n=1$. Thus $\operatorname{\pi bz}(z, a, z) \geq 0$ if and only if $a \mu\left(b, a^{\star}(b)\right) / d b \geq 0$ 


\section{REFERENCES}

Arrow, Kenneth J. "The Economics of Agency," in J.W. Pratt and R.J. Zeckhauser, eds., Principals and Agents (Cambridge, Mass.: Harvard Business School Press, 1985).

Harris, Milton and Raviv, Artur. "Optimal Incentive Contracts with Imperfect Information." Journal of Economic Theory 20 (1979): 231-259. Harris, Milton, and Raviv, Artur. "Allocation Mechanisms and the Design of Auctions," Econometrica 49 (1981): 1477-1500.

Holmstrom, Bengt. "Moral Hazard and Observability." Bell Journal of Economics 10 (1979): 74-91.

Holmstrom, Bengt., "Managerial Incentive Problems: A Dynamic Perspective," In Vetenskap och Foretagsledning (Stockholm: Swedish School of Economics and Business Administration, 1983).

Holmstrom, Bengt, and Milgrom, Paul. "Aggregation and Linearity in the Provision of Intertemporal Incentives." Working Paper No. 5, Yale School of Organization and Management, 1985.

Laffont, Jean-Jacques, and Maskin, Eric. "Optimal Reservation Price in the Vickrey Auction," Economics Letters 6 (1980): 309-313.

Laffont, Jean-Jacques, and Tirole, Jean. "Auctioning Incentive Contracts," mimeo., MIT, October 1985.

Laffont, Jean-Jacques, and Tirole Jean. "Using Cost Observation to Regulate Firms." Journal of Political Economy, to appear, 1986.

MacDonald, Glenn M. "New Directions in the Economic Theory of Agency," Ganadian Journal of Economics 17 (1984): 415-440. 
McAfee, R. Preston, and McMillan, John. "Bidding for Contracts: A PrincipalAgent Analysis." mimeo, University of Western Ontario, 1984.

Maskin, Eric, and Riley, John. "Monopoly with Incomplete Information." Rand Journal of Economics 15 (Summer 1984): 171-196.

Milgrom, Paul and Weber, Robert J. "A Theory of Auctions and Competitive Bidding." Econometrica 50: (1982): 1089-1122.

Myerson, Roger, B. "Optimal Auction Design." Mathematics of Operations Research 6 (1981): 58-73.

Myerson, Roger B. "Optimal Coordination Mechanisms in Generalized Principal-Agent Problems," Journal of Mathematical Economics 10 (1982): $67-81$.

Riley, John G. "Ex Post Information in Auctions." Working Paper No. 367 , UCLA, May 1985.

Riley, John G., and Samuelson, William. "Optimal Auctions," American Economic Review 71 (1981): 381-392.

Sappington, David. "Limited Liability Contracts between Principals and Agent." Journal of Economic Theory 29 (February 1983): 1-21. Sappington, David. "Incentive Contracting with Asymmetric and Imperfect Precontractual Knowledge." Journal of Economic Theory 34 (October 1984): $\quad 52-70$

Shavell, Steven. "Risk Sharing and Incentives in the Principal and Agent Relationship." Bell Journal of Economics 10 (1979): 55-73.

Stiglitz, Joseph E. "Incentives and Risk Sharing in Sharecropping." Review of Economic Studies 41 (April 1974): 219-255.

Weitzman, Martin L. "Efficient Incentive Contracts," Quarterly Journal of Economics 44 (June 1980): 719-730. 